\title{
Compassion-based approaches: a systematic review of their effectiveness and acceptability in schizophrenia spectrum disorders
}

\author{
Selin Mavituna ${ }^{1} \cdot$ Eric Hahn $^{2} \cdot$ Inge Hahne ${ }^{2} \cdot$ Niklas Bergmann $^{2} \cdot$ Marieke Pijnenborg $^{1} \cdot$ Thi Minh Tam Ta $^{2}$. \\ Lana Tafelski ${ }^{3} \cdot$ Kerem Böge ${ }^{2}$
}

Accepted: 11 February 2022

(c) The Author(s) 2022

\begin{abstract}
The purpose of this systematic literature review is to provide an exhaustive summary of current research to explore the prospects of compassion-based approaches in treating persons with Schizophrenia Spectrum Disorders (SSD). Thereby, studies investigating the relationship between clinical parameters and self-compassion in SSD, as well as the acceptability, feasibility, and effects of compassion-based approaches for individuals with SSD were considered. The review was performed using the Preferred Reporting Items for Systematic Reviews and Meta-Analyses guidelines and checklist. Eight studies were included for qualitative synthesis. The results indicate an important role of self-compassion for several clinical parameters, including negative associations to positive symptoms, negative symptoms, cognitive disorganization, and emotional distress. All studies reported good acceptability and feasibility. Regarding the clinical effectiveness of compassion-based approaches, a variety of clinical benefits, such as improvements of mood, affect regulation, positive symptoms, negative symptoms, cognitive disorganization, and variables relating to psychological recovery were found in individuals with SSD. It is concluded that compassion-based approaches are a promising form of intervention in the treatment of SSD. However, further research, especially randomized controlled trials, in this field is needed to understand the full potential of compassion-based approaches for individuals with SSD.
\end{abstract}

Keywords Systematic Review · Schizophrenia Spectrum Disorders · Self-Compassion · Compassion-Focused Interventions · Third Wave Cognitive Behavioural Therapy $\cdot$ Psychosis

\section{Introduction}

\section{Self-Compassion}

While compassion is a construct from Buddhist thought, Western psychology has shown a recent surge of interest in compassion (Kirby et al., 2017) which stems from the relationship between self-compassion with well-being, optimism, and happiness (Barnard \& Curry, 2011; Neff \& Germer, 2013; Zessin et al., 2015). Self-compassion involves being open and connected with one's suffering and developing the desire to alleviate symptoms and meet oneself with

\author{
Kerem Böge \\ kerem.boege@charite.de \\ Selin Mavituna \\ s.c.mavituna@student.rug.nl \\ Eric Hahn \\ eric.hahn@charite.de \\ Inge Hahne \\ inge.hahne@charite.de \\ Niklas Bergmann \\ niklas.bergmann@charite.de \\ Marieke Pijnenborg \\ g.h.m.pijnenborg@rug.nl
}

Thi Minh Tam Ta

thi-minh-tam.ta@charite.de

Lana Tafelski

1.tafelski@alumni.maastrichtuniversity.nl

1 Department of Psychology, Faculty of Behavioural and Social Science, University of Groningen, Groningen, The Netherlands

2 Department of Psychiatry and Psychotherapy, CharitéUniversitätsmedizin Berlin, Campus Benjamin Franklin, Hindenburgdamm 30, 12203 Berlin, Germany

3 Faculty of Psychology and Neuroscience, Maastricht University, Maastricht, The Netherlands 
kindness. The positive self-attitude has been operationalized along three dimensions: self-kindness, or being understanding towards oneself, as opposed to self-judgment, common humanity - or understanding one's own experience in light of a common human experience - as opposed to isolation, and mindfulness as opposed to over-identification, which entails a state of mind in which thoughts and feelings are held in balanced awareness and are decentered from instead of over-identified with (Neff, 2003a, 2003b, 2016). These dimensions interact to create a self-compassionate frame of mind (Neff, 2016; Neff \& Dahm, 2015). Self-compassion has been shown to be associated with a variety of psychological benefits. Several studies have found self-compassion to be positively correlated with positive affect and negatively correlated with negative affect (López et al., 2018; Neff et al., 2007; Sirois et al., 2015). Moreover, anxiety and depression have been shown to have a negative association with self-compassion (Muris et al., 2016). Therefore, enhancing self-compassion may be helpful to a wide array of individuals, including both clinical and non-clinical populations.

\section{Compassion-Based Approaches}

Building on the Buddhist perspective that it is possible to acquire self-compassion through mental practice, such as meditation (Jazaieri et al., 2013), compassion-based interventions have grown in interest within psychotherapy and mental health research (Gilbert, 2014; Kirby, 2016). Herein, a wide range of compassion-based approaches have been established. Among them, Compassion Focused Therapy (CFT) is the most widely studied intervention and has been shown to be acceptable and feasible in various clinical populations (Craig et al., 2020a, 2020b). As a transdiagnostic therapeutic approach, CFT is based on a neurophysiological model of emotion. The model presumes at least three emotion regulation systems, namely the threat and protection system, the drive and excitement system, and the contentment and soothing and social safeness system (Craig et al., 2020a, 2020b; Gilbert, 2005). While individuals with high self-criticism and shame show overactivity in the threat protection and drive systems, the soothing and safeness system is insufficiently accessible (Gilbert, 2009, 2014). CFT focuses on the development of the soothing and safety system, which is theorized to underpin compassion. Thereby, CFT aims to replace shame and self-criticism by developing self-compassion and mindfulness through psychoeducation, compassionate imagery, and mindfulness exercises (Braehler, Harper \& Gilbert, 2013; Gilbert, 2014). It is assumed that individuals attain emotional resistance and can learn to cope with their symptoms once they stop criticizing and shaming themselves for their symptoms, thoughts, and feelings (Braehler, Harper \& Gilbert, 2013; Gilbert, 2009). In line with this, a systematic review suggests that compassion is a promising target for intervention, particularly for individuals who experience high rates of self-criticism (Leaviss \& Uttley, 2015).

\section{Schizophrenia Spectrum Disorders (SSD)}

High levels of self-criticism and shame are typical in persons with SSD (Gerlinger et al., 2013). SSD constitute a group of severe neuropsychiatric conditions that are marked by four core features. Firstly, SSD are characterized by positive symptoms, such as hallucinations, delusions, disorganized thinking and speech, and catatonia. Secondly, SSD are also defined by negative symptoms, including diminished emotional expression, avolition, alogia, anhedonia, lack of social engagement (American Psychiatric Association, 2013; Lincoln et al., 2017), and finally, cognitive impairments (Kahn \& Keefe, 2013) and affective symptoms (Association, 2013). While negative symptoms are characterized by affective flattening and impairments in emotion perception and expression, an excess of affect is associated with acute psychotic states. Therefore, during distressing psychosis, individuals can be said to be "hypersensitive to threat" (Gumley et al., 2010a, 2010b, p. 186). Frequently, this stress reactivity is associated with stigmatization through external shame and self-criticism (Martins et al., 2020). In fact, SSD appear to be among the most stigmatized mental disorders within the general population (Mann \& Himelein, 2004; Subramaniam et al., 2017). However, stigmatizing attitudes have also been found among health professionals, such as medical students and doctors which have been associated with genetic explanations of SSD (Serafini et al., 2011). For example, one genetic liability of psychosis, or a characteristic related to the expression of a gene, is presented by neuroanatomical correlates, such as gray matter reduction in the anterior cingulate cortex (Fusar-Poli et al., 2014). While stigmatized identities have been shown to have poorer physical and mental health, poorer quality of life, and lower levels of selfesteem, self-efficacy, and hope (Gerlinger et al., 2013; Malli et al., 2016), stigmatization has been shown to mediate the relationship between compassion on and health outcomes. Additionally, negative emotional states, such as shame, selfstigma or self-criticism, have been shown to play a crucial role in shaping the content of hallucinations and delusion beliefs as positive core symptoms (Kesting \& Lincoln, 2013) and have been associated with negative symptoms of SSD (Lincoln et al., 2011). Additionally, self-criticism predicts poorer treatment outcomes (Löw et al., 2020). Therefore, reconstructing a positive sense of self is an important objective for recovery in individuals with SSD (Gumley et al., 2010a, 2010b; Waite et al., 2015).

While it has been shown that CFT is effective and well accepted in clinical populations (Craig et al., 2020a, 2020b), 
no systematic review has investigated the relationship between self-compassion, compassion-based approaches, and clinical parameters in individuals with SSD. A number of cross-sectional studies and case reports indicate a relationship between self-compassion and symptom severity for both negative (Johnson et al., 2009) and positive symptoms, as well as distress associated with symptoms (Dudley et al., 2018). Moreover, randomized controlled trials (RCTs) assessing the effectiveness of CFT in SSD are promising, especially regarding emotional recovery (Braehler, Gumley, et al., 2013; Braehler, Harper, et al., 2013). While only a few RCTs have been conducted, important trends in crosssectional data and (un)controlled trials should be examined to identify the role of self-compassion in clinical parameters in SSD and determine the potential for compassion-based interventions in this population.

Therefore, the present systematic review examines the current state of research regarding the following research questions: (1) How do clinical parameters in individuals with SSD correlate with levels of self-compassion? (2) Are compassion-based approaches feasible and acceptable for individuals with SSD? (3) What effects do compassionbased approaches have for individuals with SSD? This systematic literature review aims to provide a comprehensive synthesis of the available literature on self-compassion and its relationship to clinical parameters in individuals with SSD, as well as the effects, acceptability, and feasibility of compassion-based approaches for individuals with SSD. This is the first available systematic review concerned with compassion-based approaches for individuals with SSD, thereby providing an overview of the potential of compassion-based approaches for SSD, the current state of research of compassion-based approaches in SSD, and potential gaps in the literature. This paper, therefore, builds the foundation for an emerging area of research and clinical practice. In that, the overarching goal of this systematic review is to highlight the current state of research on compassionbased approaches for SSD and ultimately encourage future research in finding new ways to enhance recovery processes in persons with SSD.

The following paragraphs will first outline the methods, report the results and discuss them. In the final section the main conclusions of this review will be summarized.

\section{Methodology}

\section{Protocol and Registration}

The review protocol was prospectively registered in PROSPERO and was performed using the standards of the Preferred Reporting Items for Systematic Reviews and
Meta-Analyses guidelines and checklist (Moher et al., 2009). There was no funding for this systematic literature review.

\section{Search Strategy and Eligibility Criteria}

Multiple databases, namely MEDLINE, PsychINFO, PubMed, and Google Scholar were searched for English language peer-reviewed studies. Variations and combinations of the Mesh terms were used to enhance the search. Search terms included ("compass*" OR "loving kindness" OR "Compassion-focused therapy" OR "CFT" OR "mindful self-compassion" OR "MSC" OR "compassionate mind training" OR "Cultivating Compassion Training" OR "Cognitively Based Compassion Training") AND ("schizo*" OR "psychosis" OR "psychotic" OR "hallucination" OR "hearing voices" OR "delusion" OR "SSD"). Included studies had to: (1) provide a measure for (self-)compassion or use a compassion-based intervention, (2) exclusively include individuals with a SSD diagnosis, (3) include a measure of symptom severity of SSD and/or other clinical parameters, and (4) include individuals aged 18 and older. Studies were excluded if they are case reports or case series reports. As the research on compassion-based interventions for individuals with SSD is in its early stage, the entire literature base was reviewed without limiting the publication dates.

The literature search took place in February and March 2021, as well as in December 2021 by two independent raters. EBSCOhost was used for searching PsychInfo and MEDLINE simultaneously, whereas PubMed and Google Scholar were separately searched. In the first step, duplicates $(n=262)$ were removed. Afterward, titles and abstracts were screened for inclusion. The remaining articles $(n=26)$ were further assessed and judged for eligibility by reading the entire manuscript. Manual searches were conducted by scanning the reference lists of all records assessed for eligibility. No additional studies were identified, suggesting a sufficient search strategy. After removing studies based on inclusion and exclusion criteria, both raters found eight studies to be eligible for qualitative synthesis (see Fig. 1). Quality assessment using the National Institute of Health Quality Assessment Tool (https://www.nhlbi.nih.gov/health-topics/ study-quality-assessment-tools) did not indicate significant bias. Both raters independently assessed the quality of the included studies as good or fair.

\section{Results}

\section{Study Characteristics}

Study characteristics are displayed in Table 1. Eight studies published between 2011 and 2020 met the inclusion criteria for this review. The designs of these studies were as follows: 
Fig. 1 PRISMA Flow Diagram (Moher, 2009)

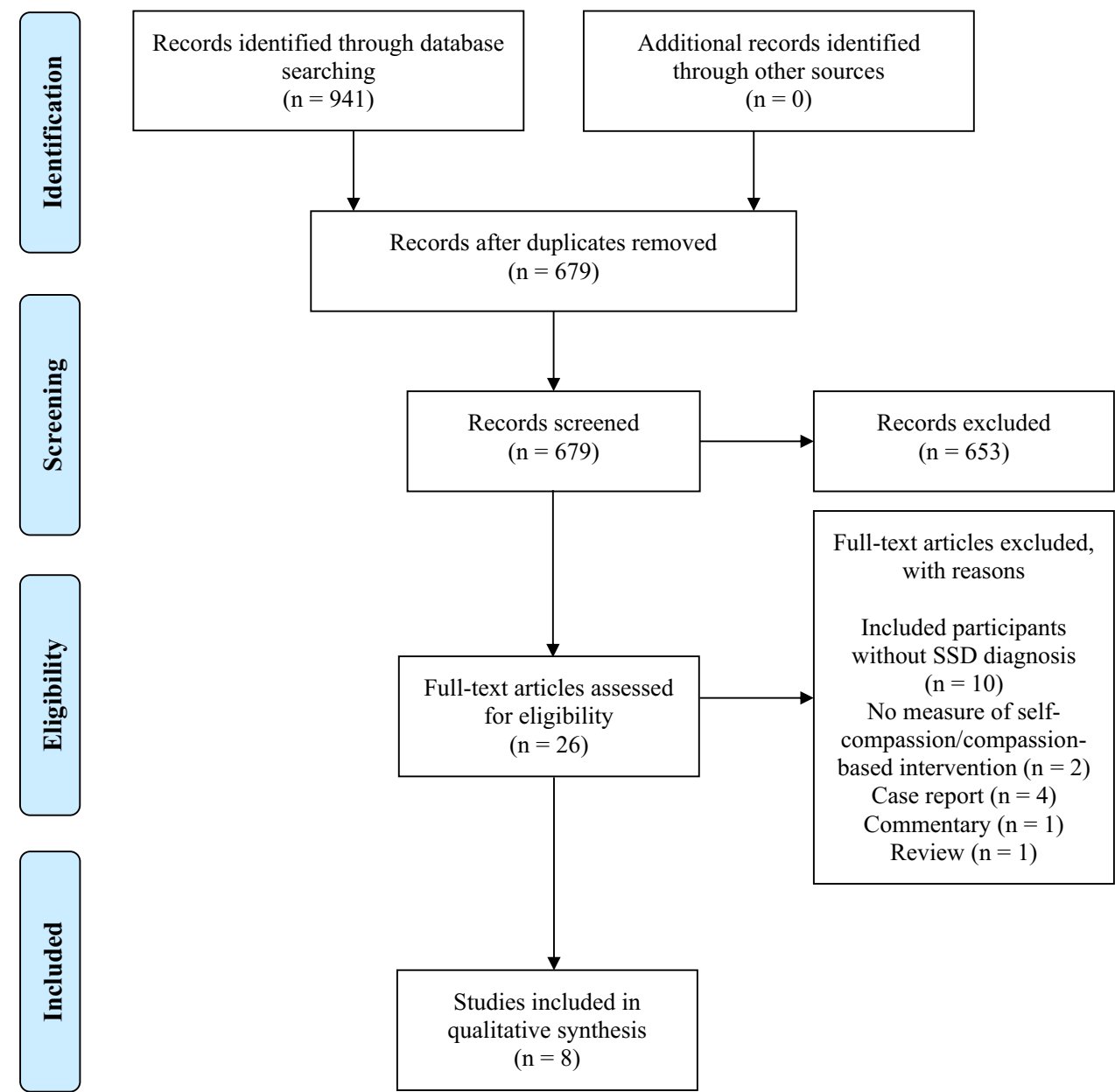

cross-sectional studies $(k=4)$, a randomized controlled trial $(k=1)$, uncontrolled prospective follow-up studies $(k=2)$, and a pre-post uncontrolled within-subject design $(k=1)$. One study was conducted in Israel, while the majority of studies were conducted in Europe and North America (Portugal, UK, USA \& Canada).

\section{Cross-Sectional Findings}

The cross-sectional studies included in this review investigated the relationship between self-compassion in individuals with SSD and a variety of clinical parameters. Two studies found that higher measures of self-compassion on the Self-Compassion Scale (SCS; Neff, 2003a) were associated with less positive symptoms as measured by the Positive and Negative Syndrome Scale (PANSS; Eicher et al., 2013; Gumley \& Macbeth, 2014; Kay et al., 1987). While Eicher et al. (2013) reported no significant relationship between self-compassion and negative symptoms, Gumley and Macbeth (2014) found greater narrative self-compassion to be associated with less negative symptoms $(r=-0.41)$ using the Narrative Compassion Interview (NCI; Macbeth, 2011).
Although no significant correlation $\left(r_{\text {range }}=-0.26\right.$ to 0.12 ; median $r=-0.14$ ) was reported between SCS and NCI, both were associated with significantly decreased scores on the cognitive disorganization $(r=-0.49, p<0.01 ; r=-0.42$, $p<0.05$, respectively) subscale of the PANSS. Additionally, with increasing scores on the NCI, decreases in the excitement subscale $(r=-0.52, p<0.01)$ were found. Here, excitement provides a measure of anger and impulsivity (Gumley \& Macbeth, 2014). Gumley and Macbeth (2014) also found a negative relationship between the SCS and emotional distress $(r=-0.51, p<0.01)$. Furthermore, scoring high on the self-kindness and mindfulness subscales of the SCS were positively associated with meaning in life as measured by the Life Regard Index (LRI; Battista \& Almond, 1973; Bercovich et al., 2020). Interestingly, when conducting a regression analysis to test the possible interaction effect of self-compassion and metacognition on meaning in life, Bercovich et al. (2020) could not find a main effect of self-compassion on meaning in life. Instead, self-compassion was found to moderate the relationship between metacognition and meaning in life. In line with this, Eicher et al. (2013) reported a negative relationship between self-compassion and insight as 


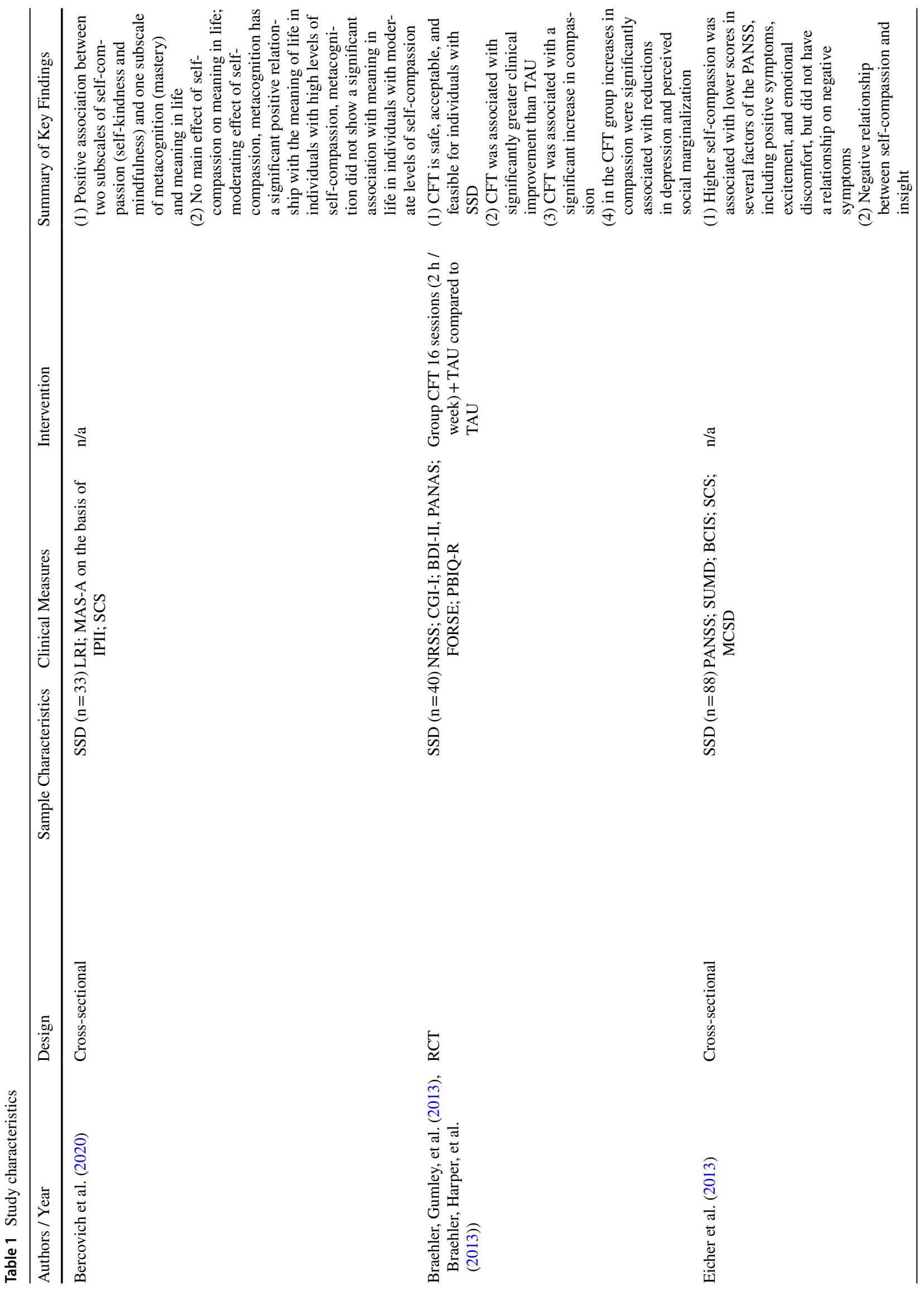




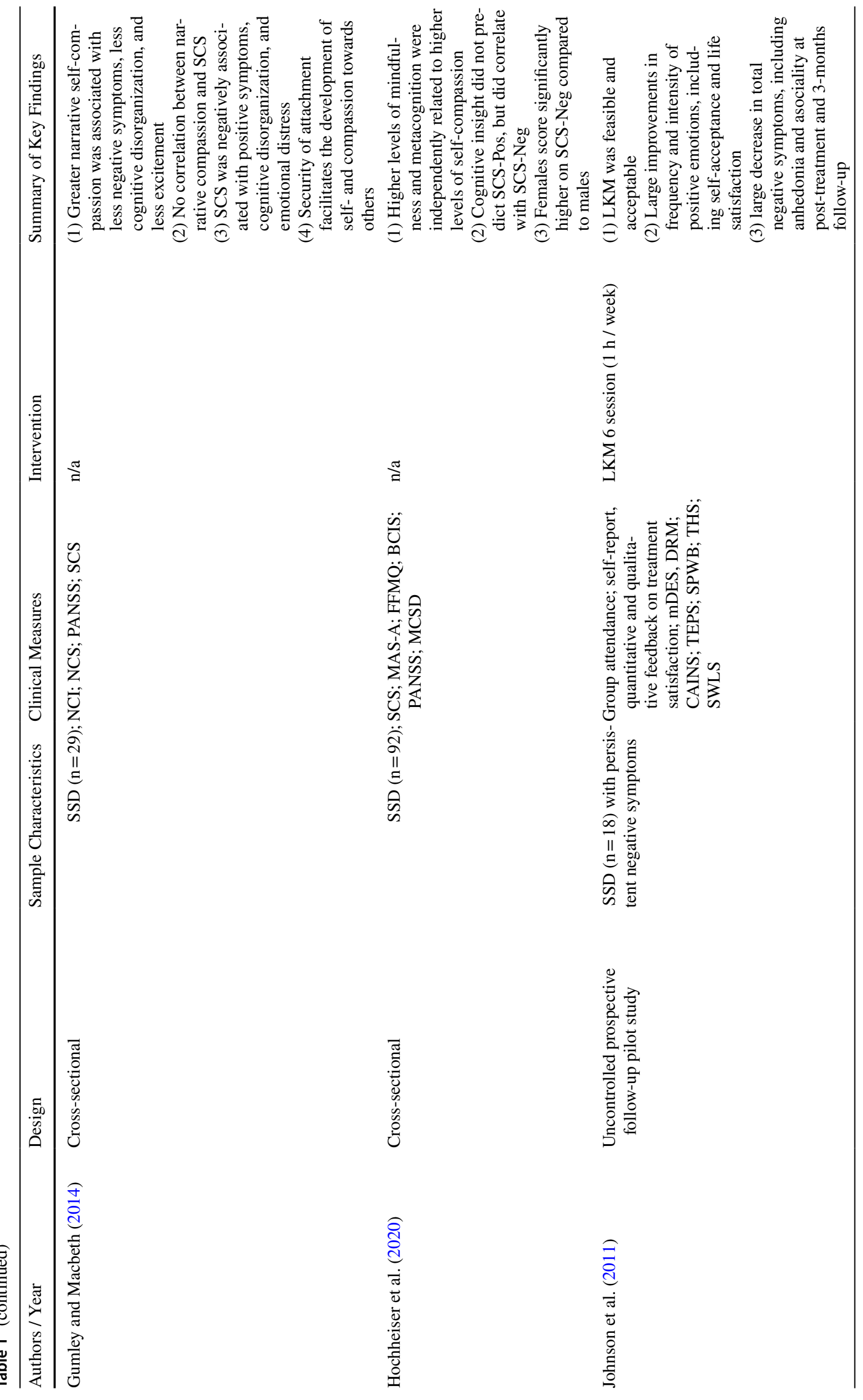




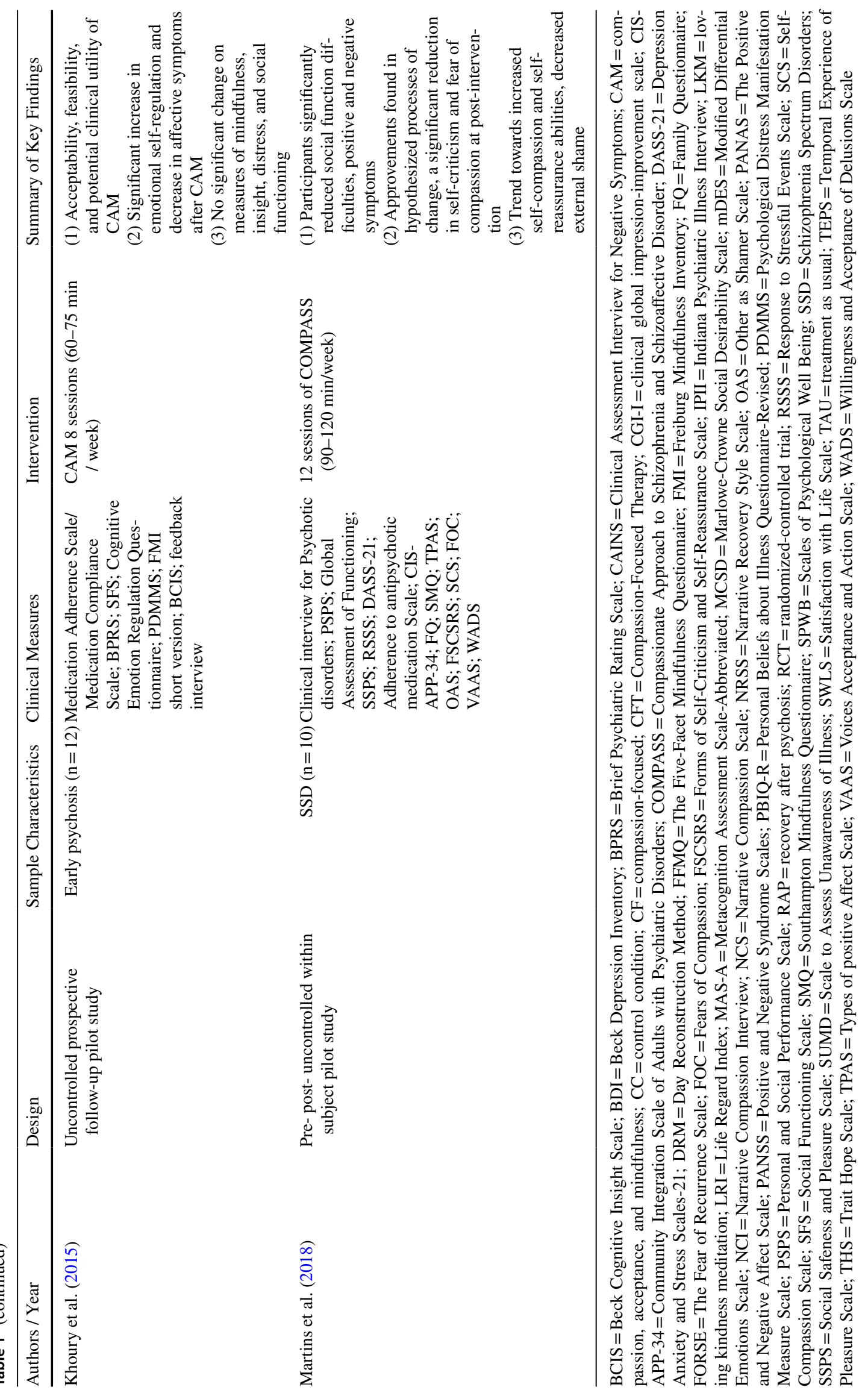


measured by the Beck Cognitive Insight Scale (Beck et al., 2004). In the same population, Hochheiser et al. (2020) suggested that cognitive insight was not related to the positively worded items of SCS but did correlate with the negatively worded items of SCS. While Hochheiser et al. (2020) found higher scores of metacognition and mindfulness to be independently related to the SCS, a non-significant negative relationship ( $r=-0.13)$ between metacognition and the total SCS score was found by Bercovich et al. (2020).

Overall, cross-sectional studies suggest self-compassion to be negatively associated with positive symptoms, negative symptoms, as well as cognitive disorganization, excitement, and emotional distress, when considering both SCS and NCI measures of self-compassion (Eicher et al., 2013; Gumley \& Macbeth, 2014). In line with this, self-compassion was positively related to meaning in life (Bercovich et al., 2020). However, mixed results have been found in the relationship between metacognition, cognitive insight, and self-compassion.

\section{Feasibility and Acceptability of Compassion-Based Interventions}

Three studies gave an indication of acceptability and feasibility of compassion-based approaches for individuals with SSD, including CFT, Loving Kindness Meditation (LKM), and a Compassion, Acceptance and Mindfulness (CAM) intervention (Braehler, Gumley, et al., 2013; Braehler, Harper, et al., 2013; Johnson et al., 2011; Khoury et al., 2015). All studies reported good acceptability and feasibility. Attendance rates were generally high, ranging between 75 and 91\% for treatment completers (Braehler et al., 2013a; Braehler, Harper, et al., 2013; Johnson et al., 2011; Khoury et al., 2015). Braehler et al., (2013a, 2013b) reported 18\% attrition from CFT (4 of 22 participants), while Johnson et al. (2011) reported 2 of 18 non-completers (11\%). CAM was completed by 12 of 17 participants (71\%; Khoury et al., 2015). Only Khoury et al. (2015) indicated the comparability between completers and non-completers of the intervention. No significant differences in sociodemographic data or any other measure were reported, except for social functioning. In fact, non-completers had significantly lower scores on the interpersonal behavior subscale $(t(15)=-2.14, p<0.05)$ of the social functioning scale (SFS; Birchwood et al., 1990), indicating that non-completers had overall lower numbers of friends, were less likely to have a partner and had lower ability to start a conversation and talk to others. Generally, participants provided positive ratings to their respective compassion-based intervention in all three studies, with participants mentioning the ease, perceived utility, relaxation, and enjoyment of their intervention (Johnson et al., 2011; Khoury et al., 2015). Additionally, participants enjoyed the social aspects and support they experienced within the intervention group (Johnson et al., 2011). While some participants experienced challenges with sending loving-kindness to all people in the world during LKM (Johnson et al., 2011) and in another participant, feelings of inadequacy and shame arose as a response to CFT, no significant distress or serious adverse reaction to the intervention were reported (Braehler et al., 2013a, 2013b; Johnson et al., 2011; Khoury et al., 2015). Overall, the evidence suggests compassionbased interventions to be safe, feasible, and acceptable.

\section{Effectiveness of Compassion-Based Interventions}

\section{Uncontrolled Pilot Studies}

Three pilot studies eligible for this systematic review used a compassion-based approach for individuals with SSD but did not include a control condition (Johnson et al., 2011; Khoury et al., 2015; Martins et al., 2018). Each of the three pilot studies included a different compassionbased approach. Khoury et al. (2015) piloted a new 8-week program for emotion regulation, combining compassion, acceptance, and mindfulness (CAM) in individuals with early psychosis, whereas Johnson et al. (2011) used LKM in 18 outpatients with SSD, focusing on cultivating a feeling of connectedness and compassion towards the self and others. Martins et al. (2018) proposed a Compassionate Approach to Schizophrenia and Schizoaffective disorder (COMPASS) which is built on a version of the emotion regulation system's model of CFT (Gilbert, 2009, 2014) that is adapted for psychosis (Gumley et al., 2010a, 2010b).

The most consistent finding across studies was the relationship between compassion-based interventions and improvements in positive affect and negative affective symptoms (Johnson et al., 2011; Khoury et al., 2015). After a 6-week program of LKM, Johnson et al. (2011) found large improvements in frequency and intensity of positive emotions, including self-acceptance and life satisfaction, both post-treatment and at 3-month follow-up. Large effect sizes were reported post-treatment for both positive emotions frequency $(d=0.78)$ and positive emotions intensity $(d=0.96)$, as well as medium effect sizes at 3-month follow-up ( $d=0.69, d=0.70$, respectively; Johnson et al., 2011). Similarly, a significant increase in emotional self-regulation and a decrease in negative affective symptoms were observed after an 8-week CAM intervention (Khoury et al., 2015). While no difference was found comparing baseline emotion regulation to post-treatment scores, moderate improvements $(d=0.61)$ were indicated on the Cognitive Emotion Regulation Questionnaire (CERQ; Garnefski \& Kraaij, 2007) at 3-month follow-up. Particularly large improvements $(d=1.00)$ were reported for variables relating to regulating negative emotions, such as self-blaming, rumination, and catastrophizing 
at 3-month follow-up. Moreover, large improvements on symptoms of anxiety $(d=0.92)$ and depression $(d=0.91)$ were observed, which were retained at 3-month follow-up, with statistically significant results for anxiety $(p<0.05)$ and approaching significance for depression $(p=0.06)$ as measured by the expanded Brief Psychiatric Rating Scale (BPRS; Khoury et al., 2015; Lukoff et al., 1986; Overall \& Gorham, 1988).

All three studies observed improvements in symptomrelated measures. Although results are mixed, significant improvements have been reported for positive symptoms, negative symptoms, and social functioning. Khoury et al. (2015) found small improvements $(d=0.25)$ on the BPRS total score at 3-month follow-up, although not statistically significant $(p=0.11)$. When investigating subscales, a small non-significant post-treatment effect was found on positive symptoms $(d=0.36)$ and negative symptoms $(d=0.40)$. Johnson et al. (2011) used the beta version of the Clinical Assessment Interview for Negative Symptoms (CAINS beta; Blanchard et al., 2011; Forbes et al., 2010) to assess negative symptoms. Analyses revealed large decreases in total negative symptoms $(d=1.68)$, as well as on the anhedonia subscale $(d=1.88)$, and medium effect size on the asociality subscale $(d=0.53)$, which were retained at 3-month follow-up. Martins et al. (2018) found preliminary significant results for positive symptoms $(Z=-2.37, p<0.05, r=-0.75)$, negative symptoms $(Z=-1.969, p<0.05, r=-0.62)$, and social functioning $(Z=-2.07, p<0.05, r=-0.65)$. However, Khoury et al. (2015) were unable to find significant improvement in social functioning $(d=-0.04, p=0.98)$.

When examining differences between those who showed improvements and those who did not, Khoury et al. (2015) reported that $50 \%$ of participants $(n=6)$ showed improvements on overall symptoms from baseline to post-treatment and 3-month follow-up. In two participants, symptoms worsened, and four participants did not show any measurable change. Participants who did not improve $(n=6)$ had significantly lower symptoms at baseline $(t(10)=-5.01, p<0.005)$. In particular, at baseline they showed lower positive symptoms, higher levels of mindfulness $(t(10)=2.84, p<0.05)$, and better social functioning $(t(10)=3.00, p<0.05)$, as compared to participants who did improve.

Moreover, there was some evidence for improvement in variables relating to psychological recovery. Participants reported increased self-acceptance $(d=0.80)$, life satisfaction $(d=0.86)$, and environmental mastery $(d=1.05)$ with large effect sizes post-treatment and moderate to large effect sizes at 3-month follow-up (Johnson et al., 2011). Similarly, Martins et al. (2018) observed significantly improved selfcriticism on the hated self subscale $(Z=-2.25, p<0.05$, $r=-0.71$ ) of the Forms of Self-Criticism and Reassurance Scale (FSCRS; Gilbert et al., 2004) after COMPASS. Moreover, Khoury et al. (2015) found large improvements after CAM on self-neglect $(d=0.71)$ and somatic concerns $(d=0.50)$, which were retained at 3-month follow-up $(p<0.05)$. No significant changes were reported for insight, distress (Khoury et al., 2015), and purpose in life (Johnson et al., 2011).

When looking at potential working mechanisms, the results are inconclusive. Johnson et al. (2011) and Khoury et al. (2015) did not include a measure of self-compassion in their study. Martins et al. (2018) did not find significant improvements in self-compassion $(\mathrm{Z}=1.68, p=0.09)$, however the observed effect size was moderate $(r=0.53)$ at post-intervention as compared to baseline measures. Khoury et al. (2015) included a measure of mindfulness but did not find significant changes in scores measured by the Freiburg Mindfulness Inventory (FMI; Buchheld et al., 2001), although the effect size at 3-month follow-up was moderate $(d=0.40)$. Nevertheless, the uncontrolled and pilot nature of the studies does not allow for the assessment of potential mediation or moderation effects.

Taken together, the evidence of the uncontrolled prospective follow-up and uncontrolled pre-post pilot studies suggests that compassion-based approaches were associated with improvements in positive affect, affect regulation, and decreased negative affect. While studies on positive and negative symptoms were less conclusive, the findings point to improvements in negative symptoms. Additionally, some evidence of significant improvement was found for variables that relate to psychological recovery. While these preliminary findings are promising, it is not clear whether self-compassion influenced the positive outcomes.

\section{Randomized Controlled Trial}

One study included in this review conducted an RCT (Braehler, Gumley, et al., 2013; Braehler, Harper, et al., 2013). In the study by Braehler et al. (2013a), Braehler, Harper, et al. (2013)), participants were randomly assigned to either group CFT in addition to treatment as usual (TAU; $n=22$ ) or TAU alone $(n=18)$. A total of 16 sessions were delivered, which were divided into three phases. The first phase included psychoeducation and explored the impact psychosis had on participants' lives. During the second phase, compassion was developed with a variety of skills, including mindfulness, imagery, attention, or appreciation. The final phase focused on expressive writing tasks to reflect compassionately on their recovery. TAU was mainly based in the community and could consist of medication, occupational therapy, psychiatric counseling, or day center support and varied among participants.

With regards to SSD symptoms, Braehler, Gumley, et al. (2013), Braehler, Harper, et al. (2013)) found a significant effect $(Z=4.04 ; p<0.001, r=-0.68)$ of CFT on the Clinical Global Impression-Improvement Scale (CGI-I; Guy, 1976). 
However, while the effects of CFT on particular symptoms remain unclear, decreases in negative affective states as measured by BDI $(Z=-2.22, p<0.05)$ were reported.

A similar trend was found using the Positive and Negative Affect Scale (PANAS), while there was a significant increase in PANAS negative affect $(r=0.47 ; p<0.05)$ in the TAU group, the CFT group showed some improvement, although not significant $(Z=-1.73, p=0.08)$.

Increases in narrative compassion were significantly correlated with reductions in depression, negative beliefs about psychosis, and fear of relapse in the CFT group. That is, Braehler, Gumley, et al. (2013), Braehler, Harper, et al. (2013)) observed a significant increase in compassion in the way CFT participants talked about their psychosis and recovery $(Z=2.43, p<0.05, r=0.42)$ using the Narrative Recovery Style Scale (NRSS; Gumley et al., 2010a, 2010b). Further analysis revealed that the increase of selfcompassion in the CFT group was significantly associated with a decrease in BDI scores $(r=0.77 ; p<0.001)$, as well as entrapment $(r=0.56 ; p<0.05)$, shame $(r=0.57 ; p<0.05)$ and social marginalization $(r=0.74 ; p<0.01)$ as measured by the Personal Belief about Illness Questionnaire-Revised (PBIQ-R; Birchwood et al., 1993). Compassion was also significantly related to the intrusiveness $(r=0.58 ; p<0.05)$ and fear of relapse $(r=0.52 ; p<0.05)$ scales of the Fear of Recurrence Scale (FORSE; Gumley \& Schwannauer, 2006). Additionally, in participants exposed to CFT, a significant association between a reduction in avoidance and a reduction in the PBIQ lack of control over illness $(r=0.57 ; p<0.05)$ was found.

Overall, the results of this RCT are promising, indicating positive effects of CFT on subjective measures of clinical improvement, negative affective states, as well as shame and social marginalization.

\section{Discussion}

The results of the present systematic review suggest three main findings. Firstly, this review found self-compassion to play an important role in several clinical parameters relevant to SSD. Consistent with findings of a meta-analysis demonstrating a negative relationship between self-compassion and psychopathology (MacBeth \& Gumley, 2012), a negative relationship was found between self-compassion and measures of symptom severity in individuals with SSD (Eicher et al., 2013; Gumley \& Macbeth, 2014). However, there are some inconsistencies in the data concerning the relationship between negative symptoms and self-compassion. While Eicher et al. (2013) observe no significant association between the SCS and negative symptoms, Gumley and Macbeth (2014) find greater narrative self-compassion associated with less negative symptoms. This may be due to the lack of correlation between the SCS - a self-report measure - and the NCI, which does not demand reflection on compassion by the participant but uses a semi-structured interview format and systematic coding system to assess self-compassion. While both NCI and SCS have good reliability, an alternative reason for this discrepancy may be differences in conceptualizations of self-compassion.

Secondly, all studies reported good acceptability and feasibility of compassion-based approaches for individuals with SSD. Given generally low attrition rates and the absence of any serious adverse effects, it can be assumed that compassion-based approaches are safe, feasible, and acceptable for individuals with SSD. This is in line with a previous systematic review, finding compassion-based approaches to be acceptable and feasible for individuals with a range of mental health problems (Craig et al., 2020a, 2020b). Therefore, this review expands the evidence contradicting the myth that interventions incorporating meditation practices are harmful to individuals with SSD (Böge, Hahne, et al., 2021; Böge, Thomas, et al., 2021). Findings show that most participants are able to increase self-compassion and integrate it as a strategy to regulate negative affect (Braehler, Gumley, et al., 2013; Braehler, Harper, et al., 2013; Johnson et al., 2011; Khoury et al., 2015). Nevertheless, one participant considered CAM to be a negative experience (Khoury et al., 2015) and another participant showed increased preoccupation with the stigma associated with having a mental health problem after CFT (Braehler et al., 2013a; Braehler, Harper, et al., 2013). However, the occurrence of adverse effects or their absence has not been consistently reported (for recommendations, see Böge, Thomas, et al., 2021; Böge et al., 2021; Ellett \& Chadwick, 2021) throughout the studies, which would have been important to identify potential vulnerability factors that discern individuals less suitable for compassion-based interventions.

And finally, with regards to the clinical effectiveness of compassion-based approaches, a variety of clinical benefits for individuals with SSD have been found. Most consistently, the results reveal a positive effect of compassion-based approaches on affect. All studies that used a compassionbased intervention and included affect measures indicate large to moderate improvements in positive affect, affect regulation and decreases in negative affect and/or affective symptoms (Braehler, Gumley, et al., 2013; Braehler, Harper, et al., 2013; Johnson et al., 2011; Khoury et al., 2015). Additionally, increases in compassion through compassion-based approaches are associated with decreases in symptoms of depression, anxiety, as well as negative beliefs about psychosis, social marginalization, fear of relapse and somatic concerns (Braehler et al., 2013a; Braehler, Harper, et al., 2013; Khoury et al., 2015). There is also evidence for improvement in variables contributing to psychological recovery, such as self-acceptance, life satisfaction, and environmental 
mastery. These results align with previous research showing self-compassion to be negatively correlated with negative self-referential processing (Mennin \& Fresco, 2013; Werner et al., 2019), thereby supporting the proposed working mechanisms of compassion-based approaches. This is comparable to findings from a previous systematic review in a variety of clinical population, indicating that compassionbased approaches show encouraging results in reducing self-criticism and shame (Craig et al., 2020a, 2020b). Since compassion-based approaches aim to alleviate negative selfreferential processing, they seem effective in individuals with SSD. Hence, these findings are in line with theoretical models underlying compassion-based approaches, conceptualizations of self-compassion, as well as previous empirical data (Barnard \& Curry, 2011; MacBeth \& Gumley, 2012).

Even though compassion-based interventions do not directly target symptom reduction, but distress associated with symptoms, findings from all four studies included in this review that used a compassion-based intervention indicate improvements in the global impression of symptoms, positive symptoms, negative symptoms, as well as improvements on social functioning (Braehler, Gumley, et al., 2013; Braehler, Harper, et al., 2013; Johnson et al., 2011; Martins et al., 2018). Khoury et al. (2015) found small but nonsignificant improvements in psychiatric symptoms, which may be due to the small sample size and the low baseline levels of positive- and negative symptoms in their sample since individuals with higher symptom severity have shown greater treatment effects, as compared to individuals with lower baseline symptoms (Khoury et al., 2015).

Some initial indications for potential mechanisms of change have been found. Of the studies using a compassionbased approach, Martins et al. (2018) and Braehler, Gumley, et al. (2013), Braehler, Harper, et al. (2013)) included a measure of self-compassion. While Martins et al. (2018) found a moderate yet non-significant improvement of selfcompassion after COMPASS compared to baseline measures, Braehler et al. (2013a), Braehler, Harper, et al. (2013)) found large significant increases in self compassion to be linked to CFT group membership. The non-significant results of Martins et al. (2018) may be due to the low number of participants $(n=10)$ and/or the pilot nature of the study. Moreover, the findings suggest that higher levels of mindfulness were associated with higher levels of self-compassion (Hochheiser et al., 2020) and moderate improvement in mindfulness at follow-up was found after CAM (Khoury et al., 2015). While mindfulness is an integral part of the conceptualization of self-compassion, it has been shown to improve clinical- and process dimensions when put in the center of interventions (Böge et al., 2020; Böge, Hahne, et al., 2021; Böge, Thomas, et al., 2021). Moreover, selfcompassion has been shown to mediate the relationship between mindfulness and symptom severity and further may be a potential mechanism of mindfulness-based interventions (Hochheiser et al., 2020; Scheibner et al., 2018; Sevel et al., 2020). From a theoretical perspective, it has been suggested that increasing mindfulness may increase awareness of distressing internal events and threatening psychotic experiences; therefore, cultivating self-compassion and acceptance may be crucial to attenuate these changes (Martins et al., 2017; Reich et al., 2021). This is in line with a meta-analysis indicating that clinical improvements were more highly correlated to compassion, acceptance, and mindfulness together rather than mindfulness alone (Khoury et al., 2013). To further understand the underlying working mechanisms, it might be useful to investigate self-compassion and mindfulness simultaneously to understand their unique and incremental effects on individuals with SSD.

The studies included in the present systematic literature review have a number of limitations owing to the early stage of research on compassion-based interventions for SSD. Several studies are in a preliminary stage, often piloting a new compassion-based approach for SSD. Therefore, sample sizes and associated power were generally small. Additionally, the three pilot studies (Johnson et al., 2011; Khoury et al., 2015; Martins et al., 2018) did not include control conditions making it challenging to attribute reported improvements to the employed intervention. Furthermore, the heterogeneity of outcome variables within the studies makes it difficult to find conclusive patterns of evidence. In order to conduct a comprehensive review of the literature, the included studies inevitably differed in terms of levels of quality. However, overall, the quality of the included studies was acceptable for the stage of research. However, reliability and validity of measures (Braehler, Gumley, et al., 2013; Braehler, Harper, et al., 2013; Eicher et al., 2013; Johnson et al., 2011; Khoury et al., 2015), as well as a priori or posthoc power calculations often remained unreported (Bercovich et al., 2020; Braehler et al., 2013a; Braehler, Harper, et al., 2013; Eicher et al., 2013; Gumley \& Macbeth, 2014; Hochheiser et al., 2020; Johnson et al., 2011; Khoury et al., 2015; Martins et al., 2018).

Aside from the limitations of the reviewed studies, the present systematic review may also encounter biases. One source of potential bias lies in the limited number of databases searched to select the articles for this systematic literature review. While only MEDLINE, PsychINFO, PubMed, and Google Scholar have been searched, some relevant publications might have been overlooked, thereby creating a skewed overview of the available findings. However, examining both published articles through scientific databases, as well as gray literature through Google Scholar, it has been tried to prevent substantial publication bias. Among the limitations of this review is also the small number of included studies which leads to a limited generalizability of the study findings. Furthermore, since only one RCT is 
included in this review, no quantitative analysis could have been conducted, which may lead to bias in the weight of certain results over others. It should also be noted that the studies included in this review were mainly conducted in Europe and North America. Therefore, a cultural bias might have been introduced in the discussed results. Nevertheless, the six studies which include sociodemographic information in terms of ethnicity and race (Braehler, Gumley, et al., 2013; Braehler, Harper, et al., 2013; Eicher et al., 2013; Gumley \& Macbeth; Hochheiser et al., 2020; Johnson et al., 2011; Khoury et al., 2015) indicate largely mixed samples, with the majority being white, of African/Caribbean descent or bi-racial, and a minority being Asian, First nation/Inuit or others.

Further studies in more diverse non-western samples are necessary to presume the generalizability of results.

Despite these limitations, this review highlights an array of clinical and research implications. The evidence suggests for potential compassion-based approaches to facilitate improvement of symptoms of SSD and promote positive affect and psychological recovery. Therefore, enhancing self-compassion may be an important step towards recovery, especially since long-lasting improvements on negative symptoms, social functioning, and quality of life are insufficiently achieved using internationally recommended treatment options (Leucht et al., 2017; Sohler et al., 2016). While the evidence suggests good acceptability and feasibility of compassion-based approaches in individuals with SSD, future research should systematically report adverse experiences. Thereby, potential vulnerability factors may be identified. Additionally, it may be useful to differentiate individuals in different stages of psychosis and accordingly develop different treatment profiles to reduce the risk of harmful treatment (Reich et al., 2021). Moreover, the reasons for the lack of correlation between different measures of selfcompassion should be investigated. To this point, it is useful to include both NCI and SCS to assess self-compassion.

Overall, this systematic review can be seen as a first step to integrate the current knowledge of and encourage future research on compassion-based approaches in SSD, as well as to target self-compassion within the therapeutic process of individuals with SSD.

\section{Conclusion}

Three main conclusions arise from this systematic review. First, self-compassion is positively associated with symptom measures, as well as variables relating to psychological recovery. Secondly, compassion-based interventions seem safe, feasible and acceptable for this population. And finally, compassion-based approaches seem to increase self-compassion and thereby improve positive affect, affect regulation, positive symptoms, negative symptoms, cognitive disorganization, and variables relating to psychological recovery. In light of these findings, it can be concluded that compassionbased approaches are a promising new direction in the treatment of SSD. Especially in consideration of their effect on affective dimensions and psychological recovery, compassion-based approaches may be an important stepping stone on the way to recovery especially for individuals with SSD demonstrating high levels of negative emotional states. In providing a comprehensive summary of the current state of research, this review lays the foundation for future research and clinical practice. However, further research, especially RCTs, in this field are needed to provide an evidence base for the full potential of compassion-based approaches in the treatment of individuals with SSD.

\section{Acknowledgements None.}

Role of Funding Source Open Access funding enabled and organized by Projekt DEAL. No funding was provided to conduct the current systematic review.

Data Availability Statement Data sharing not applicable to this article as no datasets were generated or analysed during the current study.

\section{Declarations}

Conflict of Interest There is no financial or non-financial conflict of interest among contributors.

Ethical Statement n/a.

Informed Consent n/a.

Open Access This article is licensed under a Creative Commons Attribution 4.0 International License, which permits use, sharing, adaptation, distribution and reproduction in any medium or format, as long as you give appropriate credit to the original author(s) and the source, provide a link to the Creative Commons licence, and indicate if changes were made. The images or other third party material in this article are included in the article's Creative Commons licence, unless indicated otherwise in a credit line to the material. If material is not included in the article's Creative Commons licence and your intended use is not permitted by statutory regulation or exceeds the permitted use, you will need to obtain permission directly from the copyright holder. To view a copy of this licence, visit http://creativecommons.org/licenses/by/4.0/.

\section{References}

American Psychiatric Association. (2013). Diagnostic and statistical manual of mental disorders (DSM-5®). American Psychiatric Pub.

Barnard, L. K., \& Curry, J. F. (2011). Self-compassion: Conceptualizations, correlates, \& interventions. Review of General Psychology, 15(4), 289-303. 
Battista, J., \& Almond, R. (1973). The Development of Meaning in Life. Psychiatry, 36(4), 409-427. https://doi.org/10.1080/00332 747.1973.11023774

Beck, A. T., Baruch, E., Balter, J. M., Steer, R. A., \& Warman, D. M. (2004). A new instrument for measuring insight: The Beck Cognitive Insight Scale. Schizophrenia Research, 68(2-3), 319-329. https://doi.org/10.1016/s0920-9964(03)00189-0

Bercovich, A., Goldzweig, G., Igra, L., Lavi-Rotenberg, A., Gumley, A., \& Hasson-Ohayon, I. (2020). The interactive effect of metacognition and self-compassion on predicting meaning in life among individuals with schizophrenia. Psychiatric Rehabilitation Journal, 43(4), 290-298. https://doi.org/10.1037/prj0000413 (AdvancesinPsychiatricRehabilitationFromtheStudyofMetacogniti on)

Birchwood, M., Mason, R., MacMillan, F., \& Healy, J. (1993). Depression, demoralization and control over psychotic illness: A comparison of depressed and non-depressed patients with a chronic psychosis. Psychological Medicine, 23(2), 387-395.

Birchwood, M., Smith, J., Cochrane, R., Wetton, S., \& Copestake, S. (1990). The social functioning scale the development and validation of a new scale of social adjustment for use in family intervention programmes with schizophrenic patients. The British Journal of Psychiatry, 157(6), 853-859.

Blanchard, J. J., Kring, A. M., Horan, W. P., \& Gur, R. (2011). Toward the next generation of negative symptom assessments: The collaboration to advance negative symptom assessment in schizophrenia. Schizophrenia Bulletin, 37(2), 291-299.

Böge, K., Hahne, I., Bergmann, N., Wingenfeld, K., Zierhut, M., Thomas, N., Ta, T. M. T., Bajbouj, M., \& Hahn, E. (2021a). Mindfulness-based group therapy for in-patients with schizophrenia spectrum disorders - Feasibility, acceptability, and preliminary outcomes of a rater-blinded randomized controlled trial. Schizophrenia Research, 228, 134-144. https://doi.org/10.1016/j.schres. 2020.12.008

Böge, K., Karadza, A., Fuchs, L. M., Ehlen, F., Ta, T. M. T., Thomas, N., Bajbouj, M., \& Hahn, E. (2020). Mindfulness-Based Interventions for In-Patients With Schizophrenia Spectrum Disorders-A Qualitative Approach [Original Research]. Frontiers in Psychiatry, 11(600). https://doi.org/10.3389/fpsyt.2020.00600

Böge, K., Thomas, N., \& Jacobsen, P. (2021b). Is mindfulness for psychosis harmful? Deconstructing a myth. The British Journal of Psychiatry, 218(2), 71-72. https://doi.org/10.1192/bjp.2020.165

Braehler, C., Gumley, A., Harper, J., Wallace, S., Norrie, J., \& Gilbert, P. (2013a). Exploring change processes in compassion focused therapy in psychosis: Results of a feasibility randomized controlled trial. British Journal of Clinical Psychology, 52(2), 199214. https://bpspsychub.onlinelibrary.wiley.com/doi/abs/https:// doi.org/10.1111/bjc.12009

Braehler, C., Harper, J., \& Gilbert, P. (2013b). Compassion focused group therapy for recovery after psychosis. CBT for schizophrenia: Evidence-based interventions and future directions, 236-266.

Buchheld, N., Grossman, P., \& Walach, H. (2001). Measuring mindfulness in insight meditation (Vipassana) and meditation-based psychotherapy: The development of the Freiburg Mindfulness Inventory (FMI). Journal for Meditation and Meditation Research, $1(1), 11-34$.

Craig, C., Hiskey, S., \& Spector, A. (2020a). Compassion focused therapy: A systematic review of its effectiveness and acceptability in clinical populations. Expert Review of Neurotherapeutics, 20(4), 385-400. https://doi.org/10.1080/14737175.2020.1746184

Craig, C., Hiskey, S., \& Spector, A. (2020b). Compassion Focused Therapy: a systematic review of its effectiveness and acceptability in clinical populations. Expert Review of Neurotherapeutics, 20(4), 385-400. https://doi.org/10.1080/14737175.2020b.17461 84
Donald, F., Lawrence, K. A., Broadbear, J. H., \& Rao, S. (2019). An exploration of self-compassion and self-criticism in the context of personal recovery from borderline personality disorder. Australasian Psychiatry, 27(1), 56-59. https://journals.sagepub.com/doi/ pdf/https://doi.org/10.1177/1039856218797418

Dudley, J., Eames, C., Mulligan, J., \& Fisher, N. (2018). Mindfulness of voices, self-compassion, and secure attachment in relation to the experience of hearing voices. British Journal of Clinical Psychology, 57(1), 1-17. https://doi.org/10.1111/bjc.12153

Eicher, A. C., Davis, L. W., \& Lysaker, P. H. (2013). Self-compassion: A novel link with symptoms in schizophrenia? The Journal of Nervous and Mental Disease, 201(5), 389-393. https://doi.org/ 10.1097/NMD.0b013e31828e10fa

Ellett, L., \& Chadwick, P. (2021). Recommendations for monitoring and reporting harm in mindfulness for psychosis research. The British Journal of Psychiatry, 1-3, https://doi.org/10.1192/bjp. 2021.98

Forbes, C., Blanchard, J. J., Bennett, M., Horan, W. P., Kring, A., \& Gur, R. (2010). Initial development and preliminary validation of a new negative symptom measure: The Clinical Assessment Interview for Negative Symptoms (CAINS). Schizophrenia Research, 124(1-3), 36-42.

Fusar-Poli, P., Smieskova, R., Serafini, G., Politi, P., \& Borgwardt, S. (2014). Neuroanatomical markers of genetic liability to psychosis and first episode psychosis: A voxelwise meta-analytical comparison. The World Journal of Biological Psychiatry, 15(3), 219-228.

Garnefski, N., \& Kraaij, V. (2007). The Cognitive Emotion Regulation Questionnaire: Psychometric features and prospective relationships with depression and anxiety in adults. European Journal of Psychological Assessment, 23(3), 141-149. https://doi.org/10. 1027/1015-5759.23.3.141

Gerlinger, G., Hauser, M., De Hert, M., Lacluyse, K., Wampers, M., \& Correll, C. U. (2013). Personal stigma in schizophrenia spectrum disorders: a systematic review of prevalence rates, correlates, impact and interventions. World Psychiatry, 12(2), 155-164. https://doi.org/10.1002/wps.20040

Gilbert, P. (2005). Compassion: Conceptualisations, research and use in psychotherapy. Routledge.

Gilbert, P. (2009). Introducing compassion-focused therapy. Advances in Psychiatric Treatment, 15(3), 199.

Gilbert, P. (2014). The origins and nature of compassion focused therapy. British Journal of Clinical Psychology, 53(1), 6-41.

Gilbert, P., Clarke, M., Hempel, S., Miles, J. N., \& Irons, C. (2004). Criticizing and reassuring oneself: An exploration of forms, styles and reasons in female students. British Journal of Clinical Psychology, 43(1), 31-50.

Gumley, A., Braehler, C., Laithwaite, H., MacBeth, A., \& Gilbert, P. (2010a). A compassion focused model of recovery after psychosis. International Journal of Cognitive Therapy, 3(2), 186-201.

Gumley, A., Braehler, C., Laithwaite, H., MacBeth, A., \& Gilbert, P. (2010b). Narrative recovery style scale. Unpublished manuscript.

Gumley, A., \& Macbeth, A. (2014). A pilot study exploring compassion in narratives of individuals with psychosis: Implications for an attachment-based understanding of recovery. Mental Health, Religion \& Culture, 17(8), 794-811. https://doi.org/10.1080/ 13674676.2014.922739

Guy, W. (1976). Clinical Global Impression Improvement Scale. ECDEU Assessment Manual for Psychopharmacology-Revised. Rockville, MD: US Department of Health, Education, and Welfare, Public Service, Alcohol, Drug Abuse, and Mental Health Administration, NIHM Psychopharmacology Research Branch, Division of Extramural Research Programs, 217-221.

Hochheiser, J., Lundin, N. B., \& Lysaker, P. H. (2020). The independent relationships of metacognition, mindfulness, and cognitive insight to self-compassion in schizophrenia. The Journal of Nervous and Mental Disease, 208(1), 1-6. 
Jazaieri, H., Jinpa, G. T., McGonigal, K., Rosenberg, E. L., Finkelstein, J., Simon-Thomas, E., Cullen, M., Doty, J. R., Gross, J. J., \& Goldin, P. R. (2013). Enhancing compassion: A randomized controlled trial of a compassion cultivation training program. Journal of Happiness Studies, 14(4), 1113-1126.

Johnson, D. P., Penn, D. L., Fredrickson, B. L., Kring, A. M., Meyer, P. S., Catalino, L. I., \& Brantley, M. (2011). A pilot study of lovingkindness meditation for the negative symptoms of schizophrenia. Schizophrenia Research, 129(2-3), 137-140. https://doi.org/10. 1016/j.schres.2011.02.015

Johnson, D. P., Penn, D. L., Fredrickson, B. L., Meyer, P. S., Kring, A. M., \& Brantley, M. (2009). Loving-kindness meditation to enhance recovery from negative symptoms of schizophrenia. Journal of clinical psychology, 65(5), 499-509. https://doi.org/ 10.1002/jclp.20591

Kahn, R. S., \& Keefe, R. S. (2013). Schizophrenia is a cognitive illness: time for a change in focus. JAMA psychiatry, 70(10), 1107-1112. https://doi.org/10.1001/jamapsychiatry.2013.155

Kay, S. R., Fiszbein, A., \& Opler, L. A. (1987). The positive and negative syndrome scale (PANSS) for schizophrenia. Schizophrenia Bulletin, 13(2), 261-276. https://doi.org/10.1093/schbul/13.2.261

Kesting, M.L., \& Lincoln, T. M. (2013). The relevance of self-esteem and self-schemas to persecutory delusions: a systematic review. Comprehensive psychiatry, 54(7), 766-789. https://doi.org/10. 1016/j.comppsych.2013.03.002

Khoury, B., Lecomte, T., Comtois, G., \& Nicole, L. (2015). Third-wave strategies for emotion regulation in early psychosis: A pilot study. Early Intervention in Psychiatry, 9(1), 76-83. https://doi.org/10. 1111/eip. 12095

Khoury, B., Lecomte, T., Gaudiano, B. A., \& Paquin, K. (2013). Mindfulness interventions for psychosis: A meta-analysis. Schizophrenia Research, 150(1), 176-184. https://doi.org/10.1016/j.schres. 2013.07.055

Kirby, J. N. (2016). The role of mindfulness and compassion in enhancing nurturing family environments. Clinical Psychology: Science and Practice, 23(2), 142-157.

Kirby, J. N., Tellegen, C. L., \& Steindl, S. R. (2017). A meta-analysis of compassion-based interventions: Current state of knowledge and future directions. Behavior Therapy, 48(6), 778-792. https:// doi.org/10.1016/j.beth.2017.06.003

Leaviss, J., \& Uttley, L. (2015). Psychotherapeutic benefits of compassion-focused therapy: An early systematic review. Psychological medicine, 45(5), 927-945. https://doi.org/10.1017/S003329171 4002141

Lincoln, T. M., Dollfus, S., \& Lyne, J. (2017). Current developments and challenges in the assessment of negative symptoms. Schizophrenia research, 186, 8-18. https://doi.org/10.1016/j.schres. 2016.02.035

Lincoln, T. M., Mehl, S., Kesting, M.-L., \& Rief, W. (2011). Negative Symptoms and Social Cognition: Identifying Targets for Psychological Interventions. Schizophrenia bulletin, 37(suppl_2), S23S32. https://doi.org/10.1093/schbul/sbr066

López, A., Sanderman, R., Ranchor, A. V., \& Schroevers, M. J. (2018). Compassion for others and self-compassion: Levels, correlates, and relationship with psychological well-being. Mindfulness, 9(1), 325-331. https://doi.org/10.1007/s12671-017-0777-z

Löw, C. A., Schauenburg, H., \& Dinger, U. (2020). Self-criticism and psychotherapy outcome: A systematic review and meta-analysis. Clinical Psychology Review, 75, 101808. https://doi.org/10.1016/j. cpr.2019.101808

Lukoff, D., Nuechterlein, K., \& Ventura, J. (1986). Manual for the expanded brief psychiatric rating scale. Schizophrenia Bulletin, $12,594-602$.

MacBeth, A. M. (2011). The Narrative Compassion Scale: development and validation of an interview measure of compassion and recovery in complex mental health difficulties(Doctoral dissertation, University of Glasgow).

MacBeth, A., \& Gumley, A. (2012). Exploring compassion: A metaanalysis of the association between self-compassion and psychopathology. Clinical psychology review, 32(6), 545-552. https:// doi.org/10.1016/j.cpr.2012.06.003

Malli, M. A., Forrester-Jones, R., \& Murphy, G. (2016). Stigma in youth with Tourette's syndrome: A systematic review and synthesis. European Child \& Adolescent Psychiatry, 25(2), 127-139.

Mann, C. E., \& Himelein, M. J. (2004). Factors associated with stigmatizationof persons with mental illness. Psychiatric Services, 55(2), 185-187.

Martins, M. J., Carvalho, C. B., Macedo, A., Pereira, A. T., Braehler, C., Gumley, A., \& Castilho, P. (2018). Recovery through affiliation: A compassionate approach to schizophrenia and schizoaffective disorder (COMPASS). Journal of Contextual Behavioral Science, 9, 97-102. https://doi.org/10.1016/j.jcbs.2018.07.009

Martins, M. J., Castilho, P., Santos, V., \& Gumley, A. (2017). Schizophrenia: An exploration of an acceptance, mindfulness, and compassion-based group intervention. Australian Psychologist, 52(6), 514-523. https://doi.org/10.1111/ap.12210

Martins, M. J., Macedo, A., Carvalho, C. B., Pereira, A. T., \& Castilho, P. (2020). Are shame and self-criticism the path to the pervasive effect of social stress reactivity on social functioning in psychosis? Clinical Psychology \& Psychotherapy, 27(1), $52-60$.

Mennin, D. S., \& Fresco, D. M. (2013). What, me worry and ruminate about DSM-5 and RDoC? The importance of targeting negative self-referential processing. Clinical Psychology: Science and Practice, 20(3), 258-267. https://doi.org/10.1111/cpsp.12038

Moher, D., Liberati, A., Tetzlaff, J., Altman, D. G., Group, P. (2009). Reprint-preferred reporting items for systematic reviews and meta-analyses: the PRISMA statement. Physical therapy, 89(9), 873-880.

Muris, P., Meesters, C., Pierik, A., \& de Kock, B. (2016). Good for the self: Self-compassion and other self-related constructs in relation to symptoms of anxiety and depression in non-clinical youths. Journal of Child and Family Studies, 25(2), 607-617.

Neff, K. D. (2003a). The development and validation of a scale to measure self-compassion. Self and Identity, 2(3), 223-250.

Neff, K. D. (2003b). Self-compassion: An alternative conceptualization of a healthy attitude toward oneself. Self and Identity, 2(2), 85-101.

Neff, K. D. (2016). Does self-compassion entail reduced self-judgment, isolation, and over-identification? A response to Muris, Otgaar, and Petrocchi (2016). Mindfulness, 7(3), 791-797.

Neff, K. D., \& Dahm, K. A. (2015). Self-compassion: What it is, what it does, and how it relates to mindfulness. In Handbook of mindfulness and self-regulation (pp. 121-137). Springer.

Neff, K. D., \& Germer, C. K. (2013). A pilot study and randomized controlled trial of the mindful self-compassion program. Journal of clinical psychology, 69(1), 28-44. https://doi.org/10.1002/jclp. 21923

Neff, K. D., Rude, S. S., \& Kirkpatrick, K. L. (2007). An examination of self-compassion in relation to positive psychological functioning and personality traits. Journal of Research in Personality, 41(4), 908-916. https://doi.org/10.1016/j.jrp.2006.08.002

Overall, J. E., \& Gorham, D. R. (1988). The Brief Psychiatric Rating Scale (BPRS): recent developments in ascertainment and scaling. Psychopharmacology bulletin.

Reich, D., Evans, S., Nelson, B., Hickey, T., \& O'Shea, M. (2021). Evidence Map of Mindfulness for Stages of Psychosis: State of the Literature and Implications for Future Research. Mindfulness. https://doi.org/10.1007/s12671-021-01611-x

Scheibner, H. J., Daniels, A., Guendelman, S., Utz, F., \& Bermpohl, F. (2018). Self-compassion mediates the relationship between 
mindfulness and borderline personality disorder symptoms. Journal of Personality Disorders, 32(6), 838-856.

Serafini, G., Pompili, M., Haghighat, R., Pucci, D., Pastina, M., Lester, D., Angeletti, G., Tatarelli, R., \& Girardi, P. (2011). Stigmatization of schizophrenia as perceived by nurses, medical doctors, medical students and patients. Journal of Psychiatric and Mental Health Nursing, 18(7), 576-585.

Sevel, L. S., Finn, M. T., Smith, R. M., Ryden, A. M., \& McKernan, L. C. (2020). Self-compassion in mindfulness-based stress reduction: An examination of prediction and mediation of intervention effects. Stress and Health, 36(1), 88-96. https://doi.org/10.1002/ smi.2917

Sirois, F. M., Kitner, R., \& Hirsch, J. K. (2015). Self-compassion, affect, and health-promoting behaviors. Health Psychology, 34(6), 661.

Subramaniam, M., Abdin, E., Picco, L., Pang, S., Shafie, S., Vaingankar, J., Kwok, K., Verma, K., \& Chong, S. (2017). Stigma towards people with mental disorders and its components-a perspective from multi-ethnic Singapore. Epidemiology and Psychiatric Sciences, 26(4), 371-382.
Waite, F., Knight, M. T., \& Lee, D. (2015). Self-compassion and selfcriticism in recovery in psychosis: An interpretative phenomenological analysis study. Journal of clinical psychology, 71(12), 1201-1217. https://doi.org/10.1002/jclp.22211

Werner, A. M., Tibubos, A. N., Rohrmann, S., \& Reiss, N. (2019). The clinical trait self-criticism and its relation to psychopathology: A systematic review-Update. Journal of Affective Disorders, 246, 530-547. https://doi.org/10.1016/j.jad.2018.12.069

Zessin, U., Dickhäuser, O., \& Garbade, S. (2015). The relationship between self-compassion and well-being: A meta-analysis. Applied Psychology: Health and Well-Being, 7(3), 340-364.

Publisher's note Springer Nature remains neutral with regard to jurisdictional claims in published maps and institutional affiliations. 